\title{
Traffic Peak Period Detection from an Image Processing View
}

\author{
Jianli Xiao (D), Hang Li, Xiang Wang, and Shangcao Yuan \\ Shanghai Key Lab of Modern Optical System and Engineering Research Center of Optical Instrument and System, Ministry of \\ Education, University of Shanghai for Science and Technology, No. 516 JunGong Road, Shanghai 200093, China \\ Correspondence should be addressed to Jianli Xiao; audyxiao@sjtu.edu.cn
}

Received 25 August 2017; Revised 15 December 2017; Accepted 11 January 2018; Published 15 February 2018

Academic Editor: Juan C. Cano

Copyright (C) 2018 Jianli Xiao et al. This is an open access article distributed under the Creative Commons Attribution License, which permits unrestricted use, distribution, and reproduction in any medium, provided the original work is properly cited.

Traffic peak period detection is very important for the guidance and control of traffic flow. Most common methods for traffic peak period detection are based on data analysis. They have achieved good performance. However, the detection processes are not intuitional enough. Besides that, the accuracy of these methods needs to be improved further. From an image processing view, we introduce a concept in corner detection, sharpness, to detect the traffic peak periods in this paper. The proposed method takes the traffic peak period detection problem as a salient point detection problem and uses the image processing strategies to solve this problem. Firstly, it generates a speed curve image with the speed data. With this image, the method for detection of salient points is adopted to obtain the peak point candidates. If one candidate has the lowest speed value, this candidate is the peak point. Finally, the peak period is gotten by moving forward and backward the corresponding time of the peak point with a time interval. Experimental results show that the proposed method has achieved higher accuracy. More importantly, as the proposed method solves the traffic peak period detection problem from an image processing view, it has more intuition.

\section{Introduction}

Traffic peak period detection (TPPD) has attracted more and more attentions from the researchers in intelligent transportation systems (ITS) area [1-6]. TPPD is very important for both public and traffic management departments. As for public, if the traffic peak periods have been detected, they can select the optimal travel route, which can reduce the travel time. Among traffic management departments, they can improve the ability of traffic guidance during the traffic peak periods.

The idea of TPPD is that the speed, flow, and density will achieve the peaks during the traffic peak periods [79]. As there are interrelated relations between speed, flow, and density, any one of them can be selected to detect the traffic peak periods. For example, researchers can apply the clustering methods on the speed values to detect the peak periods.

Most of the previous methods are limited to learning technologies. The clustering methods are adopted by most of the previous learning methods. Clustering methods compute the similarity of the traffic samples and assign the same labels to the samples which have the larger similarity $[10,11]$.
The metrics for similarity comparison $[12,13]$ include the Euclidean distance and city-block distance. $K$-means [14] and Fuzzy $C$-means methods [15] are the most common methods used in TPPD. Clustering methods have achieved good performances. However, their performances are greatly affected by parameter initialization, for example, the initial number of the classes and the initial centers of each class. If the parameter initialization process is reasonable, the results of the clustering methods will be good; otherwise the results will be unacceptable. This defeat will lead to unsatisfactory results in TPPD. Besides that, the TPPD processes are not intuitional. That is because there are some interleaving between different classes, unless the clustering performance is good enough.

In order to solve the problems above, we introduce a concept in corner detection, sharpness, to detect the traffic peak periods from an image processing view. The proposed method takes the traffic peak period detection problem as a salient point detection problem and uses the image processing strategies to solve this problem. Firstly, it employs the speed values of one road to generate a speed curve image. Then, the salient point detection method is applied 


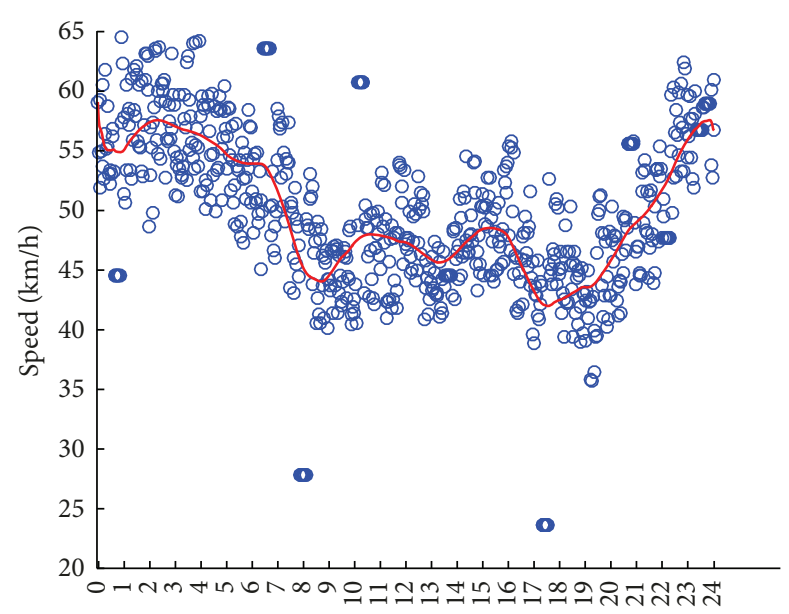

Time (h)

Figure 1: Traffic speed data and its smooth curve.

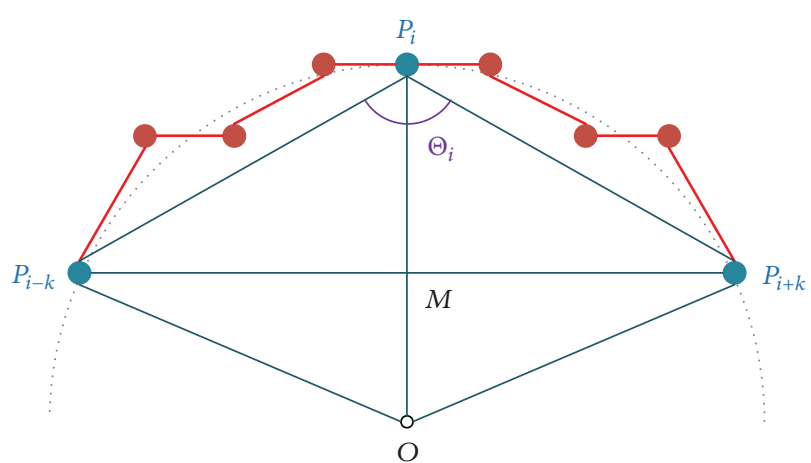

FIGURE 2: The enlarged view of one part of the speed curve.

on this image to obtain the peak point candidates. For all the candidates, if one candidate has the lowest speed value, this candidate is the peak point. The peak period is gotten by taking the corresponding time of the peak point as the center and moving forward and backward a time interval. Extensive experiments indicate that the proposed method has achieved higher accuracy. More importantly, as the proposed method solves the traffic peak period detection problem from an image processing view, it is very intuitional and easy to be carried out.

The remaining parts of this paper are structured as follows. Section 2 describes the proposed method in detail. Section 3 is devoted to empirical results. Finally, conclusions have been drawn in Section 4.

\section{Proposed Method}

2.1. Data Preprocessing. In order to achieve better performance, the raw traffic data afforded by Shanghai Transportation Information Center should be preprocessed first. The methods for noise elimination [16-18] and missing values completion $[19,20]$ are adopted to improve the quality of the traffic data. Good data quality is the guarantee of good TPPD performance. In this research, the linear interpolation method [21] is used to complete the missing traffic data, and the average smoothing method [22] is introduced to eliminate the noise data. With these two steps, the traffic data quality is improved greatly. Then, the traffic data is used to draw the speed curve, and a speed curve image is generated, which is shown in Figure 1. In this figure, the circles denote the traffic samples. Namely, they are speed values at different times. The solid line is the smoothed speed curve.

2.2. Construct Peak Point Candidate Sets. In Section 2.1, the speed curve image is obtained. In this section, we will present the method for generating the peak point candidate sets by introducing the sharpness concept $[23,24]$.

According to [23], we present the definition of sharpness and use it to capture the peak point candidates. As shown in Figure 2, the solid points denote the points in the speed curve. Suppose we select one point from the speed curve and denote it as $P_{i}$. Take $P_{i}$ as the center, and move forward and backward $k$ points distance, respectively. Then, two points are obtained, and we denote them as $P_{i+k}$ and $P_{i-k}$. With three points $P_{i-k}, P_{i}$, and $P_{i+k}$, we can do circle fitting and use the dotted line to represent the arc. $O$ is the center of the circle. $\Theta_{i}$ is the angle between the lines $P_{i} P_{i-k}$ and $P_{i} P_{i+k}$. According to experience, the integer $k$ is selected from [3 10] 
for good detection results. As $k$ is small enough, we can let $\left|P_{i-k} P_{i}\right|=\left|P_{i} P_{i+k}\right|$, approximately. Namely, $\triangle P_{i-k} P_{i} P_{i+k}$ is an isosceles triangle. Meanwhile, $O$ is the center of the circle. Thus, $P_{i} O \perp P_{i-k} P_{i+k} . \triangle P_{i-k} P_{i} M$ and $\triangle P_{i+k} P_{i} M$ are both right triangles.

In $\triangle P_{i-k} P_{i} M$, we have

$$
\sin \left(\angle P_{i-k} P_{i} M\right)=\sin \left(\frac{\Theta_{i}}{2}\right)=\frac{\left|P_{i-k} M\right|}{\left|P_{i-k} P_{i}\right|} .
$$

Similarly, in $\triangle P_{i+k} P_{i} M$, we have

$$
\sin \left(\angle P_{i+k} P_{i} M\right)=\sin \left(\frac{\Theta_{i}}{2}\right)=\frac{\left|P_{i+k} M\right|}{\left|P_{i+k} P_{i}\right|} .
$$

Combine (1) and (2); we can get

$$
\sin \left(\frac{\Theta_{i}}{2}\right)=\frac{\left|P_{i-k} M\right|}{\left|P_{i-k} P_{i}\right|}=\frac{\left|P_{i+k} M\right|}{\left|P_{i+k} P_{i}\right|} .
$$

From (3), the following formula can be derived:

$$
\sin \left(\frac{\Theta_{i}}{2}\right)=\frac{\left|P_{i-k} P_{i+k}\right|}{\left|P_{i-k} P_{i}\right|+\left|P_{i} P_{i+k}\right|} .
$$

According to (4), we can define a sharpness variable with the following formula:

$$
\operatorname{sharp}_{i}=1-\sin \left(\frac{\Theta_{i}}{2}\right)=1-\frac{\left|P_{i-k} P_{i+k}\right|}{\left|P_{i-k} P_{i}\right|+\left|P_{i} P_{i+k}\right|},
$$

where $\operatorname{sharp}_{i}$ is the sharpness value of the $i$ th point in the speed curve.

With (5), the sharpness values of each point in the speed curve can be computed. The threshold $T$ of the sharpness can be computed as

$$
T=\frac{\sum_{i=1}^{m} \operatorname{sharp}_{i}}{m},
$$

where $m$ is the total number of nonzero elements of the sharpness values.

As the morning and evening peaks appear in certain periods. If we set the start time and end time manually for the morning and evening peaks according to the experience, the traffic samples which do not lie in these two periods are not the peak point candidates. For example, if we estimate the periods for morning and evening peaks appeared are, respectively, [7:00 10:00] and [16:00 20:00], only the traffic samples in these two periods can be peak point candidates. Suppose the estimated morning and evening peak periods are $R_{\mathrm{am}}$ and $R_{\mathrm{pm}}$. The rule for judging whether the ith point is a peak point candidate is

$$
f\left(\operatorname{sharp}_{i}\right)= \begin{cases}1 & \text { if } \operatorname{sharp}_{i} \geq T, t_{i} \in\left\{R_{\mathrm{am}}, R_{\mathrm{pm}}\right\} \\ 0 & \text { else }\end{cases}
$$

where $f\left(\operatorname{sharp}_{i}\right)$ is a discriminant function and $t_{i}$ is the sampling time of the $i$ th traffic sample. If $f\left(\operatorname{sharp}_{i}\right)=1$, the $i$ th point is a peak point candidate; otherwise, the $i$ th point is not a peak point candidate.

With (7), we can judge whether one point in the speed curve is a peak point candidate. Namely, when the sharpness value of one point in the speed curve is not smaller than the sharpness threshold $T$ and $t_{i}$ is in the morning peak period or the evening peak period, this point is a peak point candidate. Following this rule, we can obtain two peak point candidate sets: morning peak point candidate set and evening peak point candidate set.

2.3. Capture the Peak Points. In Section 2.2, the morning and evening peak point candidate sets are obtained. Now, we sort the elements in the morning and evening peak point candidate sets according to their sharpness values from the maximum to the minimum, respectively. Then, we select top $l$ samples. In these $l$ traffic samples, if one has the smallest speed value, this traffic sample is a peak point, and the corresponding time is the peak time. With this idea, the morning peak time and evening peak time are detected.

For example, we sort the traffic samples in the estimated morning (evening) peak periods. Then, we select top 9 samples and show them in Figures 3(a) and 3(b). At 08:58, the speed is the smallest. Thus, this traffic sample is the peak point in the morning, which can be seen in Figure 3(c). With the same method, we can get that the evening peak time is $17: 34$.

2.4. Compute the Traffic Peak Periods. The peak time is obtained in Section 2.3. In this subsection, we will describe the method for computing the peak periods.

In real applications, the peak periods are more important for the traffic guidance and control. The peak period is gotten by taking the corresponding time of the peak point as the center and moving forward and backward a time interval. And, the time interval is estimated manually according to the experience. In our research, the time interval is 40 minutes. For example, in Figure 3(c), the morning peak time is 08:58. According to this method, the morning peak period can be computed as [08:18 09:48]. With the same method, we can also compute the evening peak period, which is [16:54 18:14].

As a summary, we present the framework of the proposed method in Figure 4, which affords an overview of our method.

\section{Experiments}

3.1. Dataset. In order to evaluate the performance of the proposed method, extensive experiments are performed with the GPS dataset, which is provided by Shanghai Transportation Information Center. The whole GPS data in one day is taken to perform the experiments, which contains road section ID, the longitude and latitude coordinates of the center points of each road section, the speed of each road section, and the traffic state of each road section. The GPS data is sampled from the whole road sections in Shanghai, which includes 14786073 traffic samples. The sample time and the speed values of the road sections are adopted to 


\begin{tabular}{|c|c|c|c|c|c|}
\hline Time (AM) & Speed & Sharpness & Time (PM) & Speed & Sharpness \\
\hline 08:08 & 33.6047 & $0.0177^{\uparrow}$ & $17: 34$ & 29.9806 & $0.1160 \uparrow$ \\
\hline 08:10 & 33.5202 & 0.0174 & $17: 32$ & 29.9987 & 0.1103 \\
\hline 08:06 & 33.7077 & 0.0156 & $17: 36$ & 29.9891 & 0.1098 \\
\hline 08:12 & 33.4497 & 0.0150 & $17: 30$ & 30.0427 & 0.0864 \\
\hline 08:04 & 33.8285 & 0.0124 & $17: 38$ & 30.0189 & 0.0685 \\
\hline 09:00 & 33.1149 & 0.0118 & $17: 28$ & 30.1109 & 0.0563 \\
\hline 09:02 & 33.1567 & 0.0116 & $17: 40$ & 30.0641 & 0.0398 \\
\hline 08:14 & 33.3899 & 0.0114 & $17: 26$ & 30.2011 & 0.0313 \\
\hline 08:58 & 33.0813 & 0.0110 & $16: 10$ & 36.0124 & 0.0216 \\
\hline
\end{tabular}

(a)

(b)

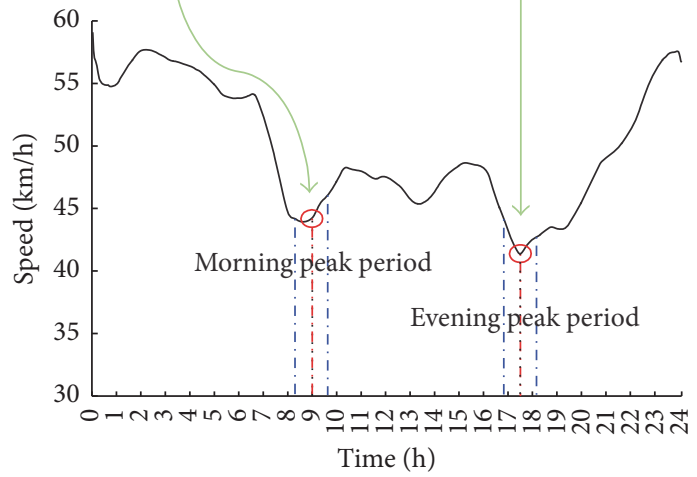

(c)

FIGURE 3: The peak points in the speed curve: (a) sorted traffic samples in the estimated morning peak period; (b) sorted traffic samples in the estimated evening peak period; (c) two peak points on the speed curve.

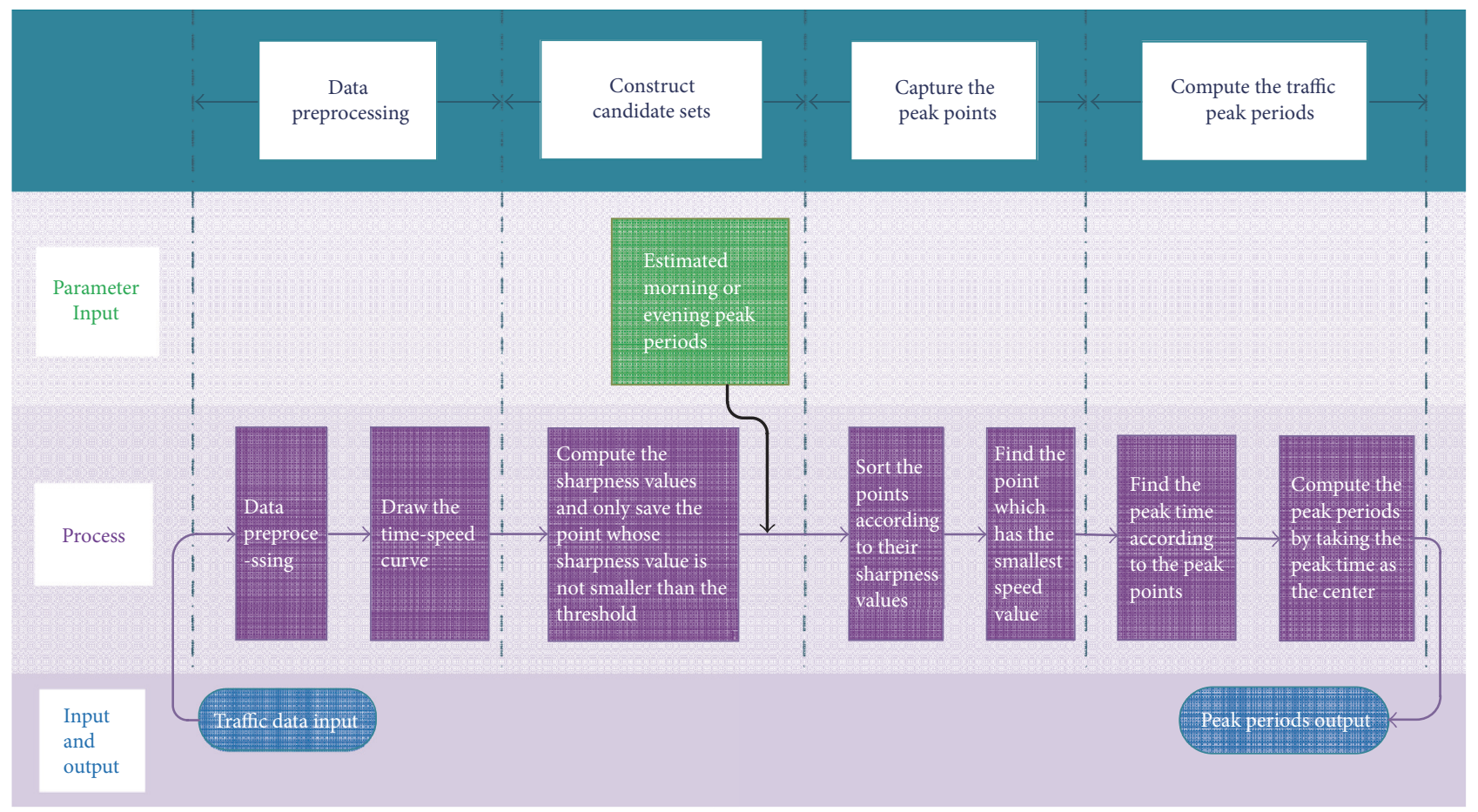

FIGURE 4: The framework of the proposed method. 


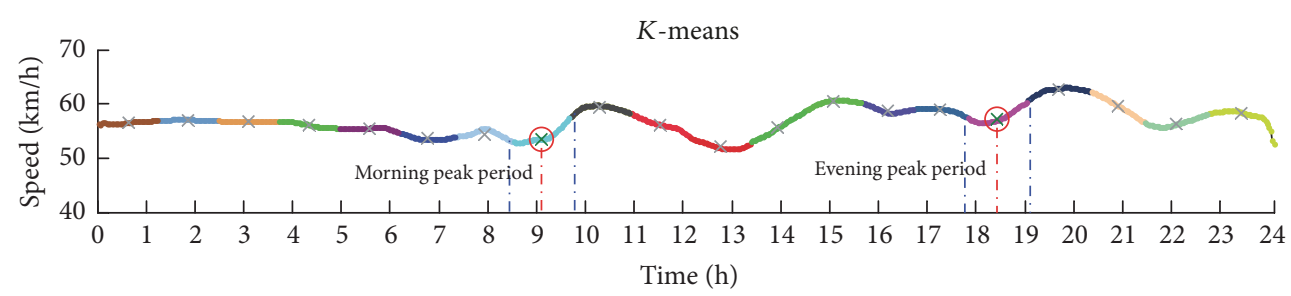

(a)

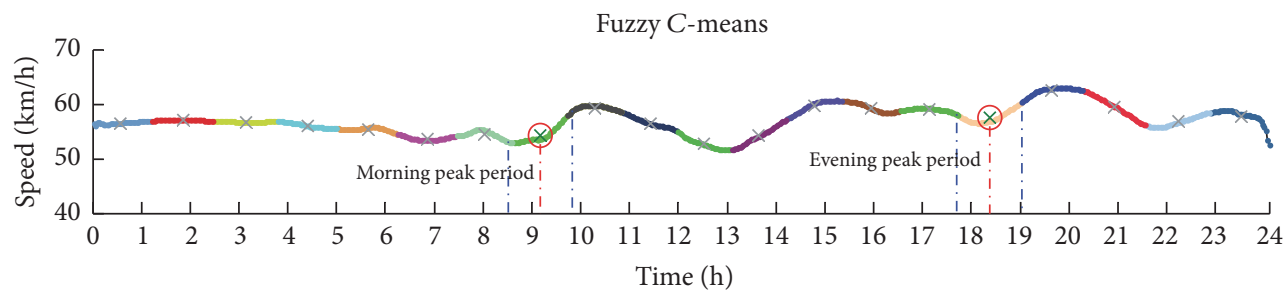

(b)

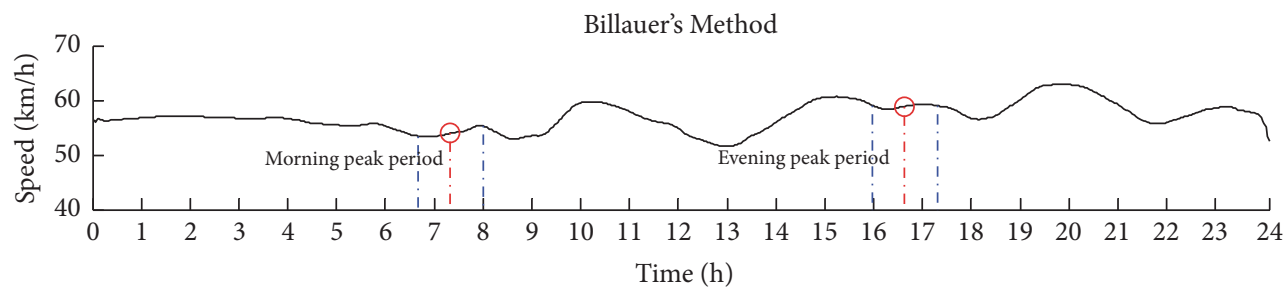

(c)

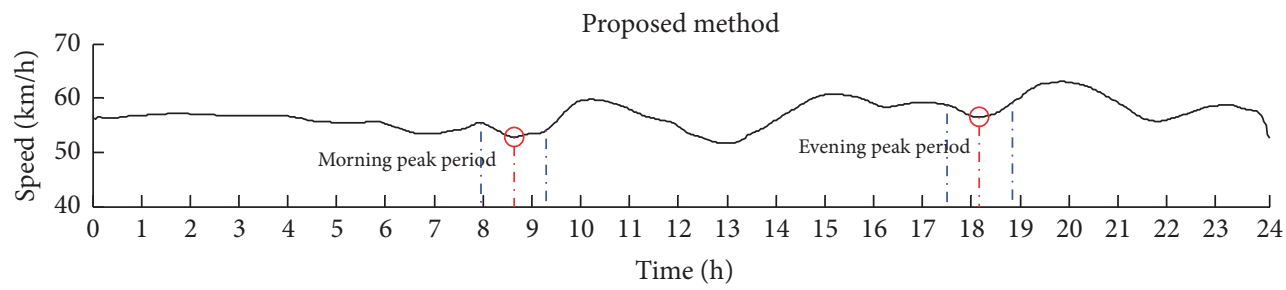

(d)

Figure 5: The detection results on Road 5.

perform the experiments. For comparison, $K$-means [14] and Fuzzy $C$-means methods [15] are also employed to perform the experiments. As the peak detection methods $[25,26]$ are commonly used to detect the traffic peak periods. A peak detection method, Billauer's method [26], is adopted to perform the experiments at the same time. The true values of the morning and evening peak periods are calibrated manually.

3.2. Results and Discussions. Due to limitations on space, some parts of detection results are shown in Figures 5-7. In these figures, the points marked with "o" are the peak points. The corresponding time of the peak point is presented with a point on the $x$-axis. The red dashed line between this point and the peak point is perpendicular to the $x$-axis. The blue dashed line at the left of the red dashed line has a crossover point with the the $x$-axis, and this crossover point denotes the start time of the peak period. Also, we can find that there is a crossover point between the right blue dashed line and the $x$-axis, which denotes the end time of the peak period. The curves in Figures 5(a), 5(b), 6(a), 6(b), 7(a), and 7(b) present the detection results of $K$-means and Fuzzy $C$-means. For these two curves, different colors denote different classes, and " $x$ " is the center of one class. Each curve has two circles. The left and right circles represent the morning and evening peak points, respectively.

Figures 5-7 show that the proposed method has achieved higher accuracy and robustness compared with $K$-means method, Fuzzy $C$-means method, and Billauer's method. In Figure 5, $K$-means method and Fuzzy $C$-means method have big errors in detecting the evening peak periods. And, Billauer's method has detected the morning and evening peak periods faultily. Figure 6 shows that $K$-means method has big error in detecting the morning peak period. It also indicates that Fuzzy $C$-means method and Billauer's method have detected the evening peak period faultily. From Figure 7, we can see that $K$-means method, Fuzzy $C$-means method, and Billauer's method generate big errors in the evening peak period detection. 


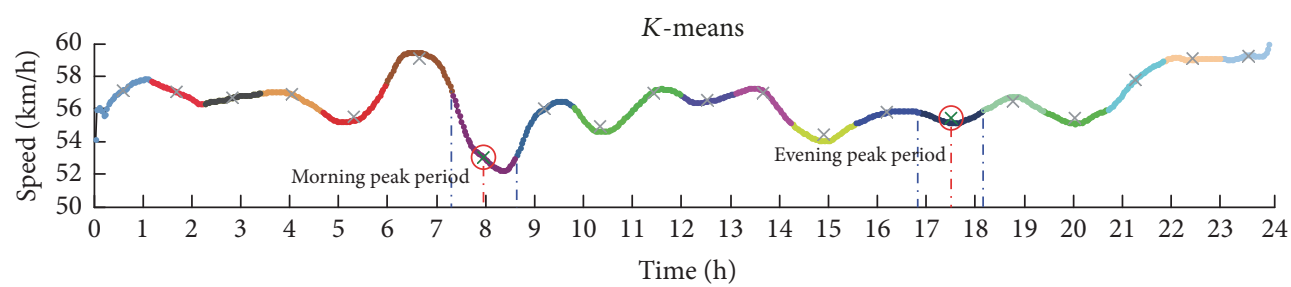

(a)

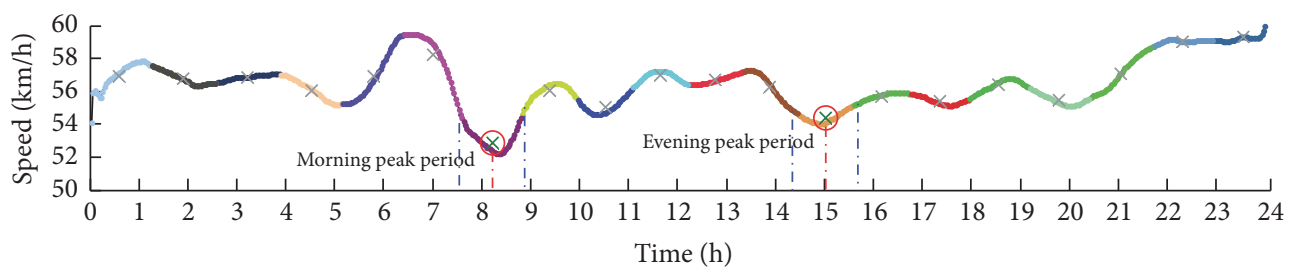

(b)

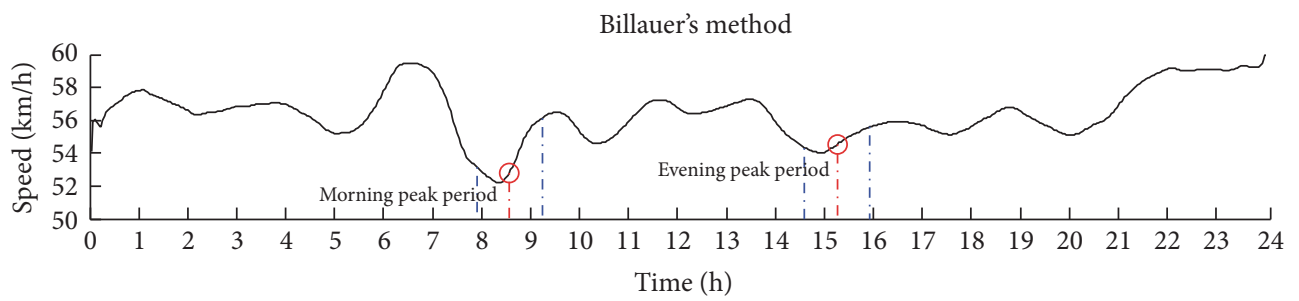

(c)

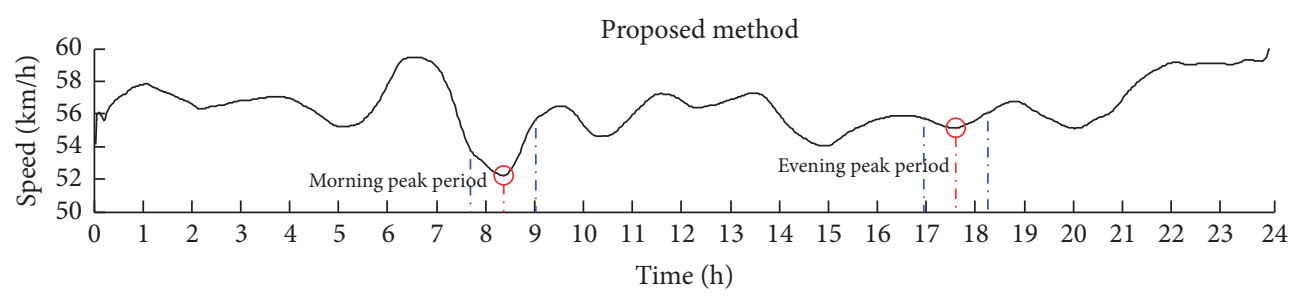

(d)

FIgURE 6: The detection results on Road 6.

As the accuracy of the four methods is decided by the peak times they detected. We present the true vales of the peak times in one figure, which are marked with “*”, and we also present the peak times each method detected in the same figure. All peak times are presented in minutes. For example, if the peak time is 625, it denotes 10:25. Figures 8 and 9 , respectively, show the results in detecting the morning and evening peak time. In these two figures, the curves of each method are generated by connecting the peak points with line segments. Thus, the curve more close to the true values (namely, the asterisks) has higher detection accuracy. From Figures 8 and 9, we can see that the curve of the proposed method is the most close to the true values, which indicates that the proposed method has the highest accuracy and its performance is the most robust.

\section{Conclusions}

In this research, we propose a new method to detect the traffic peak period. Different from the classical methods, the proposed method is from an image processing view, which introduces a concept in corner detection, sharpness, to detect the traffic peak period. It employs the speed values of one road to generate a speed curve image. With this image, the methods for detection of salient points in image processing are adopted to find the peak point candidates. If one candidate has the lowest speed value, this candidate is the peak point. The peak period is gotten by taking the corresponding time of the peak point as the center and moving forward and backward a time interval. Extensive experiments indicate that the proposed method has a higher accuracy. More importantly, as the proposed method solves the traffic peak period detection problem from an image processing view, it has more intuition. We believe that this method can be easily applied on the traffic peak period detection.

\section{Conflicts of Interest}

The authors declare that they have no conflicts of interest. 


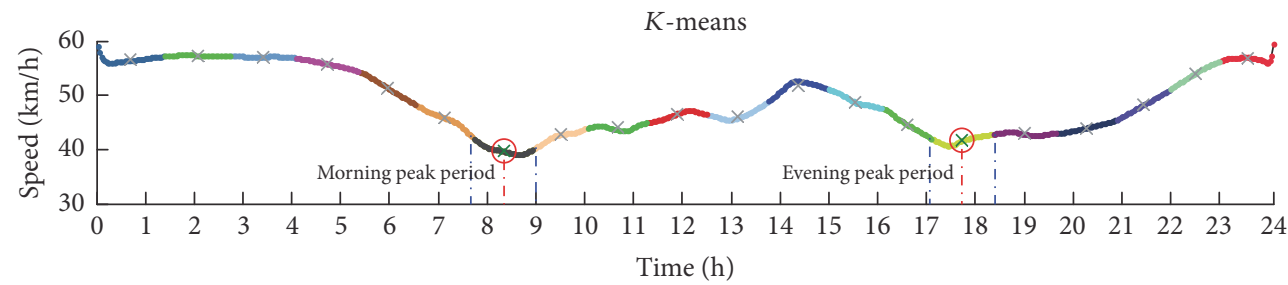

(a)

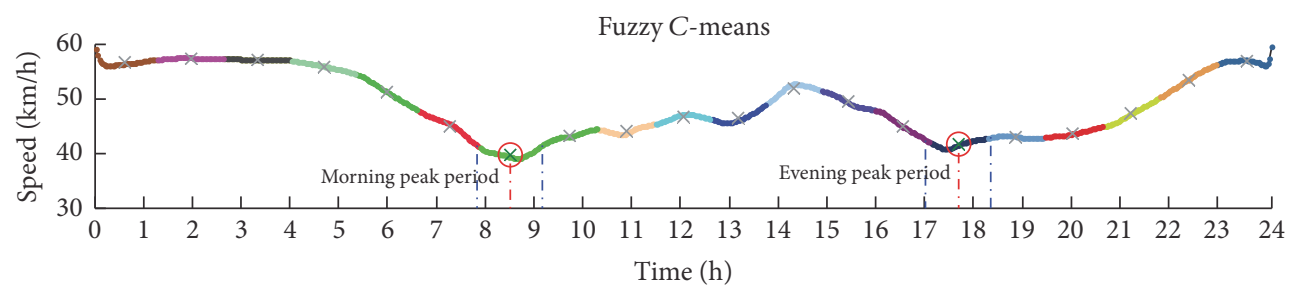

(b)

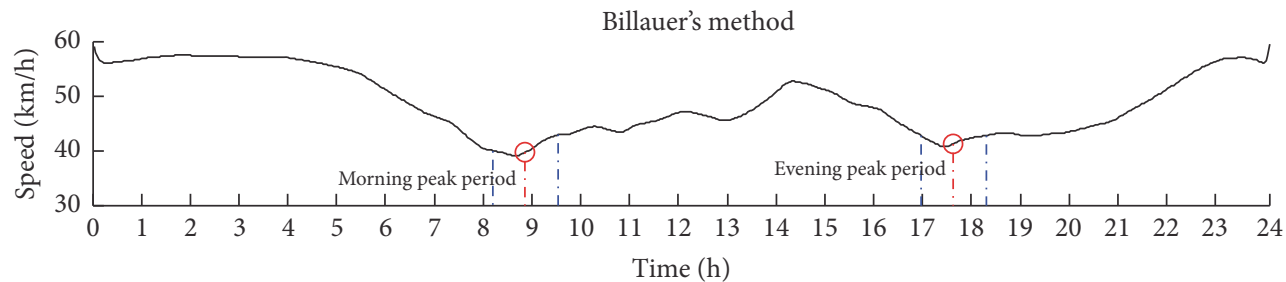

(c)

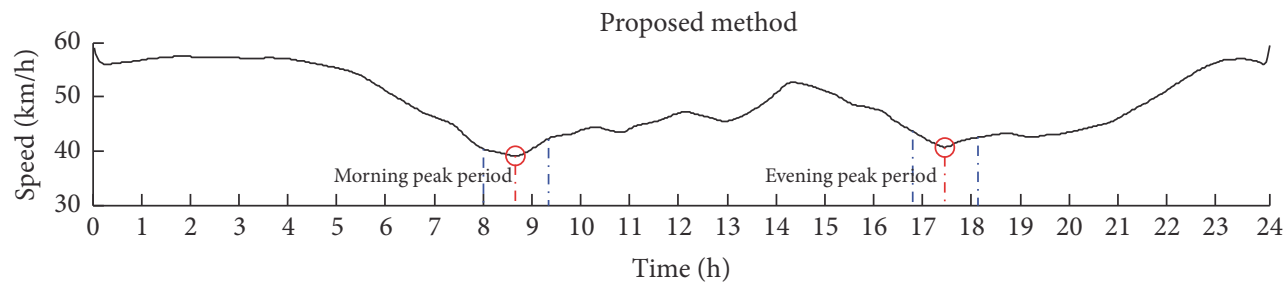

(d)

FIgURE 7: The detection results on Road 8.

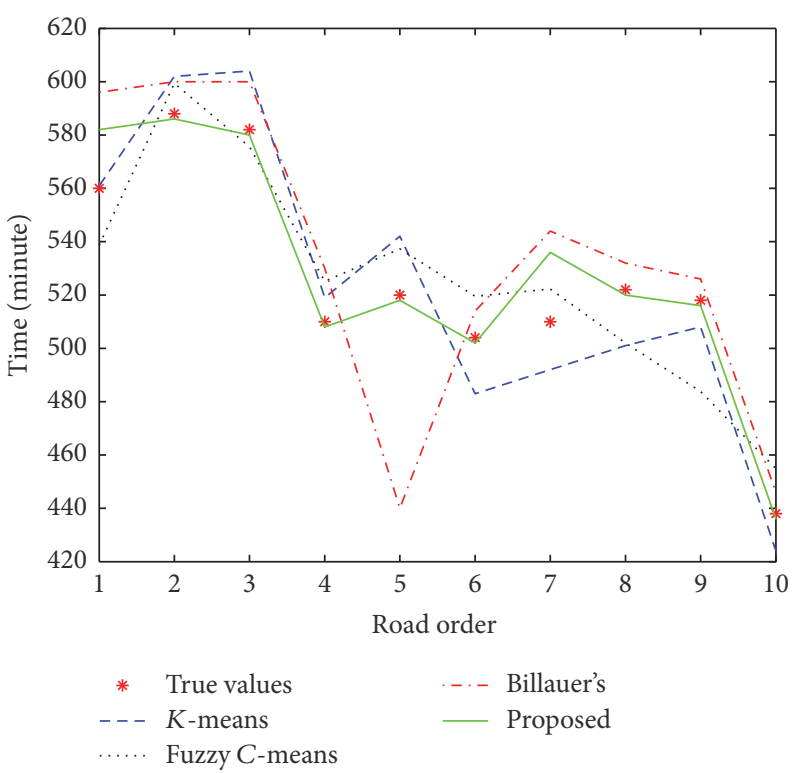

FIgURE 8: Performance comparison of four methods for morning peak period detection. 


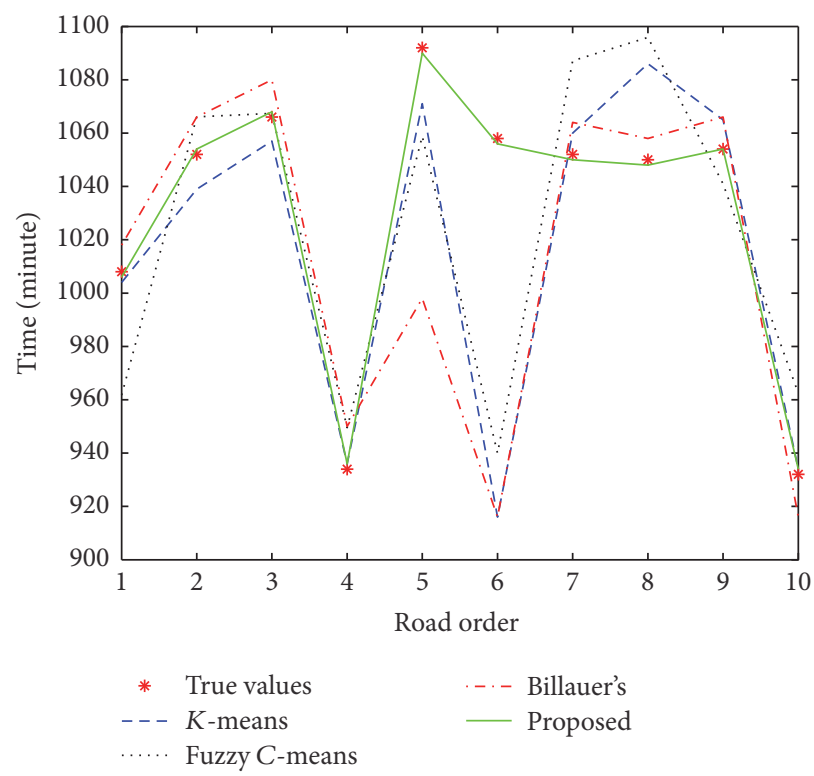

FIGURE 9: Performance comparison of four methods for evening peak period detection.

\section{Acknowledgments}

This work is supported by China NSFC Program under Grant no. 61603257. The authors appreciate Shanghai Transportation Information Center very much for providing the raw traffic data.

\section{Supplementary Materials}

In order to show the traffic peak period detection results of the proposed method well, a video demo is provided. It presents the framework of the software coded based on our paper. Also, the usage of this software can be learned from this video. (Supplementary Materials)

\section{References}

[1] V. Kumar, C. R. Bhat, R. M. Pendyala, D. You, E. Ben-Elia, and D. Ettema, "Impacts of incentive-based intervention on peak period traffic: experience from the Netherlands," Transportation Research Record, vol. 2543, pp. 166-175, 2016.

[2] Y. Cheng, X. Ye, and Z. Wang, "A forecasting model of the proportion of peak-period boardings for urban mass transit system: a case study of osaka prefecture," in in. Transportation Research Board 95th Annual Meeting. No. 16-3782, pp. 16-3782, 2016.

[3] S. Hankey and G. Lindsey, "Facility-demand models of peak period pedestrian and bicycle traffic: comparison of fully specified and reduced-form models," Transportation Research Record, vol. 2586, pp. 48-58, 2016.

[4] A. T. M. Oakil, L. Nijland, and M. Dijst, "Rush hour commuting in the Netherlands: gender-specific household activities and personal attitudes towards responsibility sharing," Travel Behaviour and Society, vol. 4, pp. 79-87, 2016.

[5] L. A. Kasper, "Differentiating the transnational from the national in a multicultural setting: Identity in persepolis and rush hour," The Kennesaw Journal of Undergraduate Research, vol. 4, no. 1, p. 1, 2016.

[6] S. S. Pulugurtha and S. S. Nambisan, "Estimating time dependent O-D trip tables during peak periods," Journal of Advanced Transportation, vol. 34, no. 3, pp. 369-389, 2000.

[7] T. Nagatani, "Effect of stopover on motion of two competing elevators in peak traffic," Physica A: Statistical Mechanics and its Applications, vol. 444, pp. 613-621, 2016.

[8] J. Xiao, X. Gao, Q.-J. Kong, and Y. Liu, "More robust and better: a multiple kernel support vector machine ensemble approach for traffic incident detection," Journal of Advanced Transportation, vol. 48, no. 7, pp. 858-875, 2014.

[9] J. Xiao and Y. Liu, "Traffic incident detection using multiplekernel support vector machine," in Transportation Research Record: Journal of the Transportation Research Board, No. 2324, pp. 44-52, Transportation Research Board of the National Academies, Wash, USA, 2012.

[10] V. Novák, I. Perfilieva, and A. Dvorák, "Fuzzy cluster analysis," in Insight into Fuzzy Modeling, pp. 137-148, 2016.

[11] E. Elhamifar and R. Vidal, "Sparse subspace clustering: algorithm, theory, and applications," IEEE Transactions on Pattern Analysis and Machine Intelligence, vol. 35, no. 11, pp. 2765-2781, 2013.

[12] L. Zhang, L. Zhang, X. Mou, and D. Zhang, "FSIM: a feature similarity index for image quality assessment," IEEE Transactions on Image Processing, vol. 20, no. 8, pp. 2378-2386, 2011.

[13] P. Mitra, C. A. Murthy, and S. K. Pal, "Unsupervised feature selection using feature similarity," IEEE Transactions on Pattern Analysis and Machine Intelligence, vol. 24, no. 3, pp. 301-312, 2002.

[14] S. Gupta, R. Kumar, K. Lu, B. Moseley, and S. Vassilvitskii, "Local search methods for k-means with outliers," in Proceedings of the 43rd International Conference on Very Large Data Bases, VLDB 2017, pp. 757-768, New Delhi, India, September 2017. 
[15] E. Esme and B. Karlik, "Fuzzy c-means based support vector machines classifier for perfume recognition," Applied Soft Computing, vol. 46, pp. 452-458, 2016.

[16] S. Verbaeten and A. Van Assche, "Ensemble Methods for Noise Elimination in Classification Problems," in International Workshop on Multiple Classifier Systems, vol. 2709 of Lecture Notes in Computer Science, pp. 317-325, Springer Berlin Heidelberg, Berlin, Germany, 2003.

[17] K. Y. Sanliturk and O. Cakar, "Noise elimination from measured frequency response functions," Mechanical Systems and Signal Processing, vol. 19, no. 3, pp. 615-631, 2005.

[18] J. R. Mou, S. M. R. Islam, X. Huang, and K. L. Ou, "A new approach of noise elimination methodology for ECG signal," in Proceedings of the 2017 International Conference on Electrical, Computer and Communication Engineering, ECCE 2017, pp. 921-927, Bangladesh, February 2017.

[19] Q.-Y. Liu, L. Zhu, D.-S. Huang et al., "Visual data completion via local sensitive low rank tensor learning," in Proceedings of the 2016 International Joint Conference on Neural Networks, IJCNN 2016, pp. 2693-2698, Canada, July 2016.

[20] W. Ruan, P. Xu, Q. Z. Sheng, N. J. Falkner, X. Li, and W. E. Zhang, "Recovering Missing Values from Corrupted Spatio-Temporal Sensory Data via Robust Low-Rank Tensor Completion," in International Conference on Database Systems for Advanced Applications, vol. 10177 of Lecture Notes in Computer Science, pp. 607-622, Springer International Publishing, New York, NY, USA, 2017.

[21] C. Chen, F. Liu, Y. Li, C. Yan, and G. Liu, "A robust interpolation method for constructing digital elevation models from remote sensing data," Geomorphology, vol. 268, pp. 275-287, 2016.

[22] A. F. De Araujo, C. E. Constantinou, and J. M. R. S. Tavares, "Smoothing of ultrasound images using a new selective average filter," Expert Systems with Applications, vol. 60, pp. 96-106, 2016.

[23] J. Xiao, Z. Xiang, B. Wang, and Y. Liu, "Adaptive algorithm for corner detecting based on the degree of sharpness of the contour," Optical Engineering, vol. 50, no. 4, Article ID 047008, 2011.

[24] J. Xiao, W. Xiang, and Y. Liu, "Vehicle logo recognition by weighted multiclass support vector machine ensembles based on sharpness histogram features," IET Image Processing, vol. 9, no. 7, pp. 527-534, 2015.

[25] F. Scholkmann, J. Boss, and M. Wolf, "An efficient algorithm for automatic peak detection in noisy periodic and quasi-periodic signals," Algorithms, vol. 5, no. 4, pp. 588-603, 2012.

[26] E. Billauer, "Peak detection using matlab," Eli Billauers home page, 2012, http://billauer. co. il/peakdet. html. 


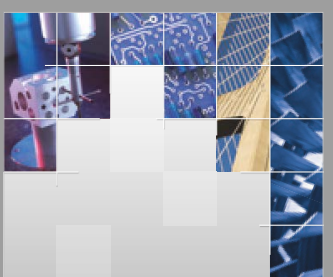

\section{Enfincering}
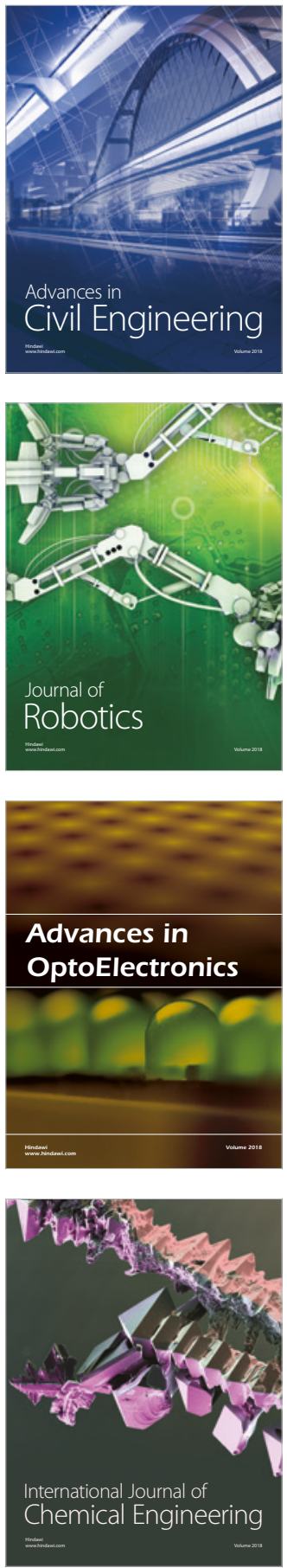

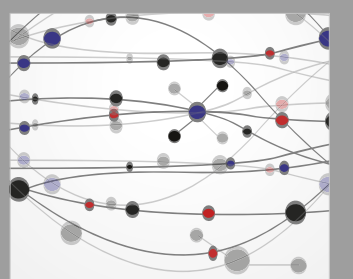

\section{Rotating \\ Machinery}

The Scientific World Journal

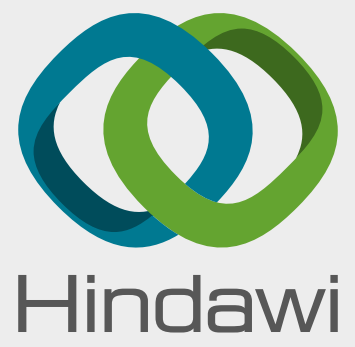

Submit your manuscripts at

www.hindawi.com
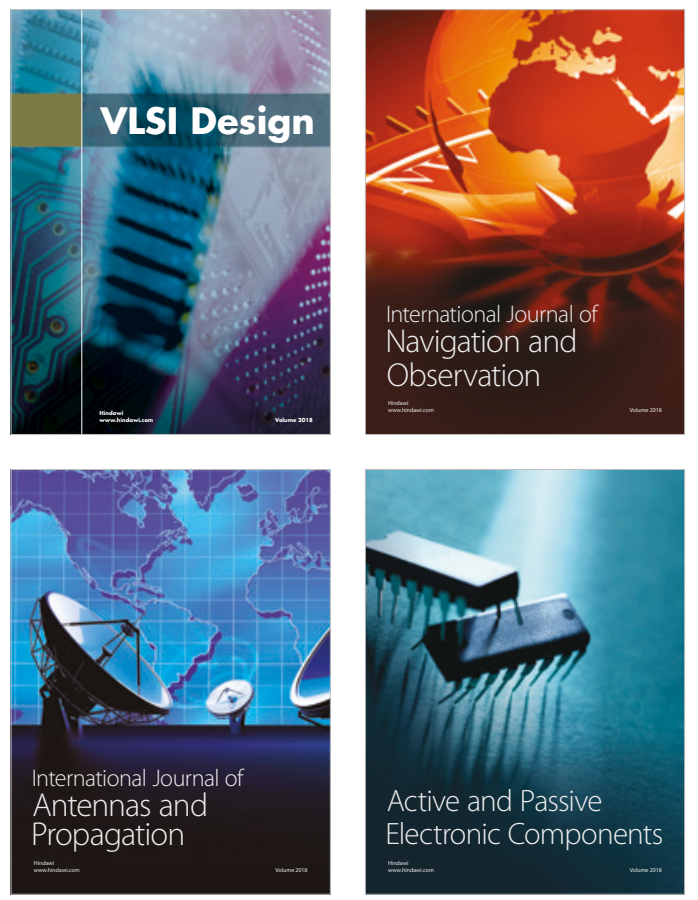
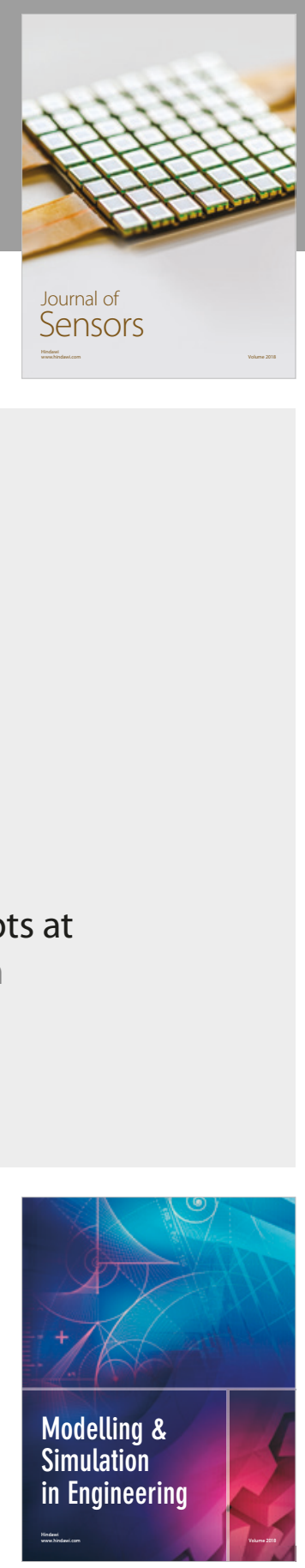

\section{Advances \\ Multimedia}
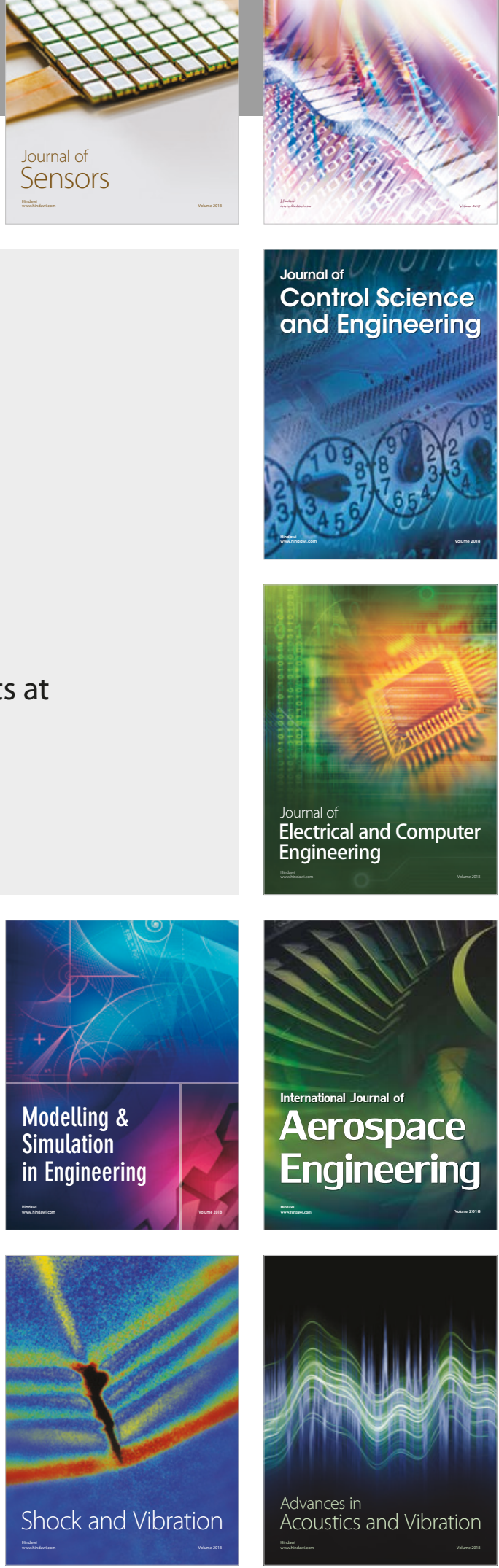